\title{
Basic performance and future developments of BeiDou global navigation satellite system
}

\author{
Yuanxi Yang (10, Yue Mao and Bijiao Sun
}

\begin{abstract}
The core performance elements of global navigation satellite system include availability, continuity, integrity and accuracy, all of which are particularly important for the developing BeiDou global navigation satellite system (BDS3). This paper describes the basic performance of BDS-3 and suggests some methods to improve the positioning, navigation and timing (PNT) service. The precision of the BDS-3 post-processing orbit can reach centimeter level, the average satellite clock offset uncertainty of 18 medium circular orbit satellites is $1.55 \mathrm{~ns}$ and the average signal-inspace ranging error is approximately $0.474 \mathrm{~m}$. The future possible improvements for the BeiDou navigation system are also discussed. It is suggested to increase the orbital inclination of the inclined geostationary orbit (IGSO) satellites to improve the PNT service in the Arctic region. The IGSO satellite can perform part of the geostationary orbit (GEO) satellite's functions to solve the southern occlusion problem of the GEO satellite service in the northern hemisphere (namely the "south wall effect"). The space-borne inertial navigation system could be used to realize continuous orbit determination during satellite maneuver. In addition, high-accuracy space-borne hydrogen clock or cesium clock can be used to maintain the time system in the autonomous navigation mode, and stability of spatial datum. Furthermore, the ionospheric delay correction model of BDS-3 for all signals should be unified to avoid user confusion and improve positioning accuracy. Finally, to overcome the vulnerability of satellite navigation system, the comprehensive and resilient PNT infrastructures are proposed for the future seamless PNT services.
\end{abstract}

Keywords: BDS-3, PNT, Performance, Constellation, lonospheric delay

\section{Introduction}

The BeiDou navigation satellite system (BDS) provides more services compared with other global navigation satellite systems (GNSSs). The BeiDou global navigation satellite system, (BDS-3), is the third step of China's satellite navigation system construction $[1,2]$. In addition to the positioning, navigation and timing (PNT) service provided by all GNSSs, BDS-3 also provides regional message communication (1000 Chinese characters per time) and global short message communication (40 Chinese characters per time), global search and rescue service (SAR), regional precise point positioning (PPP) service, embedded satellite-based augmentation service (BDSBAS), and space environment monitoring function [3-5].

*Correspondence: yuanxi_yang@163.com

State Key Laboratory of Geo-Information Engineering, Xi'an 710054, China
By the end of 2019, 28 BDS-3 satellites had been successfully launched, including 24 medium circular orbit (MEO) satellites, 3 inclined geostationary orbit (IGSO) satellites and 1 geostationary orbit (GEO) satellite. However, only $18 \mathrm{MEO}$ satellites can provide services thus far, and other satellites are in test phase.

The BDS-3 baseline system with 18 MEO satellites has begun providing initial services to global users on December 27, 2018, and at least five satellites are visible for global users. Many scholars have described the BDS-3 status and the main service function indexes [2-6], including the constellation design, service type, navigation signal system, space-time datum and orbit determination method of BDS-3 system, etc. The spacetime datum, signal-in-space quality, accuracy of satellite broadcast ephemeris, the accuracy of the post-processing precise orbit, time synchronization accuracy, satellite clock offset accuracy and the basic PNT service 
performance of the BDS-3 baseline system have be calculated and analyzed [3-5, 7-10]. Because BDS-3 satellites are equipped with inter-satellite links (ISL), more research achievements have been made in the fields of ISL supported determination of satellite orbit and clock offset [3-5, 11-13]. According to the preliminary calculation results, the ranging accuracy of the ISLs of BDS-3 is about $4 \mathrm{~cm}$; if the satellite orbits are determined using only regional station observations, the three-dimensional orbit accuracy of the overlapping arc is about $60 \mathrm{~cm}$; with ISL measurements, the orbit accuracy is about $30 \mathrm{~cm}$, the 24-h orbit prediction accuracy is also raised from 140 to $51 \mathrm{~cm} \mathrm{[3-5],} \mathrm{and} \mathrm{the} \mathrm{radial} \mathrm{accuracy} \mathrm{can} \mathrm{reach} 10 \mathrm{~cm}$, as evaluated through laser observations.

The performance of BDS-3 presented by different scholars, using different data sources, are highly similar to each other. From the aspect of signal, the ratio bias of signal component effective power is better than $0.25 \mathrm{~dB}$, the $\mathrm{S}$ curve bias is less than 0.3 , the signal-in-space accuracy calculated with post-processing ephemeris and broadcast ephemeris is approximately $0.5 \mathrm{~m}$ (root mean square, RMS), and the signal-in-space continuity and availability are approximately $99.99 \%$ and $99.78 \%$, respectively [7]. The timing accuracy is better than $19.1 \mathrm{~ns}$ (95\%), which satisfies the system service performance specifications. Compared with BDS-2, BDS-3 exhibits significant improvement in system coverage, spatial signal accuracy, availability and continuity [2-5]. At the user's end, service improvements such as the BDS-3 signal design and optimal receiving mode [14], precision orbit determination $[3-5,9,15]$, and precise point positioning technology [16] have been reported in relevant literature and will not be repeated herein.

The main performance evaluation methods and formulae are given in the second section. The current performance of BDS-3 by the end of August 2019 is presented and analyzed in the third section. Under normal circumstances, the main performance indicators provided by BDS-3 can satisfy or surpass the design indicators, which are comparable to the service performance of other GNSSs. The possible improvement strategies for BDS-3 are presented in the fourth section from the aspects of system construction, satellite constellation, satellite payload, etc. Conclusions are presented in the final section of the paper.

\section{Performance evaluation methods}

The spatial signal accuracies of BDS-3 include broadcast orbit accuracy, broadcast clock offset accuracy, signal-inspace ranging error (SISRE), broadcast ionospheric delay model accuracy, etc. Specific evaluation methods are as follows:
Broadcast orbit accuracy: the post precise satellite positions are taken as references, and the accuracy of the positions calculated by the broadcast ephemeris is the evaluation object. The root mean square error (RMSE) of the broadcast orbit is evaluated using the differences (errors of the broadcast orbit) of the broadcast and precise satellite orbits in the same coordinate system, i.e. China Geodetic Coordinate System 2000 (CGCS2000) [17] and the same time scale, i.e. BDS time (BDT) [18]. The orbit error can be obtained using the following formula [19-22]

$$
\Delta \vec{R}=\left(\vec{R}_{b r d}+A \cdot P C O_{b r d}\right)-\vec{R}_{p r e}
$$

where $\Delta \vec{R}$ is the broadcast orbit error vector; $\vec{R}_{b r d}$ is the satellite position vector calculated by the broadcast ephemeris with the unit $\mathrm{m}$; $A$ denotes the transformation matrix from the satellite-fixed coordinate system to the earth fixed system; $P C O_{b r d}$ is the correction from the satellite antenna phase center to the satellite mass center with the unit m; $\vec{R}_{\text {pre }}$ is the precise satellite position vector calculated by precise ephemeris with the unit $\mathrm{m}$. And then the broadcast orbit accuracy in a specific period can be obtained from the statistics of differences of the satellite positions.

Broadcast clock offset accuracy: the post precise satellite clock offsets are taken as the references, and the accuracy of the satellite clock offsets calculated by the broadcast ephemeris is the evaluation object. The RMS of the broadcast clock offset is evaluated using the differences between the broadcast and precise clock offsets. The broadcast clock offset is calculated using the following formula

$$
\Delta \tilde{c}_{k}^{t}=\Delta c_{k}^{t}-\frac{\sum_{k=1}^{N} \Delta c_{k}^{t}}{N}
$$

where $\Delta \tilde{c}_{k}^{t}$ is the broadcast clock offset of the satellite $k$ after subtracting the time datum difference at the epoch $t ; \Delta c_{k}^{t}$ is the difference between the broadcast and precise clock offsets after time group delay (TGD) and antenna phase center corrections; $N$ denotes the number of satellites; $k$ denotes the satellite number; $t$ denotes the epoch time. $\Delta c_{k}^{t}$ is calculated using the following formula

$$
c_{k}^{t}=B_{k}^{t}-\frac{T G D_{\mathrm{m} 1} f_{\mathrm{m} 1}^{2}-T G D_{\mathrm{m} 2} f_{\mathrm{m} 2}^{2}}{f_{\mathrm{m} 1}^{2}-f_{\mathrm{m} 2}^{2}}-\frac{e_{k}^{p r e}-e_{k}^{b r d}}{c}-\bar{C}_{k}^{t}
$$

where $B_{k}^{t}$ is the clock offset of the $k$-th satellite calculated by the broadcast clock offset parameters at the epoch $\mathrm{t}$; $\bar{C}_{k}^{t}$ is the precise clock offset of the $k$-th satellite at the epoch $\mathrm{t}$ with the unit $s ; \frac{T G D_{\mathrm{m} 1} f_{\mathrm{m} 1}^{2}-T G D_{\mathrm{m} 2} f_{\mathrm{m} 2}^{2}}{f_{\mathrm{m} 1}^{2}-f_{\mathrm{m} 2}^{2}}$ is the TGD correction of the satellite clock offset obtained using the broadcast TGD parameters with the unit $s ; T G D_{m 1}$ is the time delay difference of on-board equipment 
corresponding to the $f_{m 1}$ frequency signal with unit $s ; f_{m 1}$ and $f_{m 2}$ denote the signal carrier frequencies adopted in the double differential ionosphere-free combination; $\frac{e_{k}^{p r e}-e_{k}^{\text {ord }}}{c}$ is the difference between the antenna phase center corrections of the broadcast and precise clock offsets with the unit $s ; e_{k}^{p r e}$ and $e_{k}^{b r d}$ denote the $\mathrm{Z}$ components of the satellite antenna phase center corrections adopted by the products of the precise clock offset and the broadcast clock offset respectively with the unit $\mathrm{m}$. The accuracy of broadcast clock offset in the evaluation period can then be obtained through the statistical calculation of clock offset errors.

Signal-in-space ranging error (SISRE): the post orbit parameters are taken as the reference, and the evaluation object is set to be the projection accuracy of the combined error of the satellite orbit error and the clock offset on the sightline between the satellite and the user. It is usually calculated using the differences of the broadcast and post processed precise orbit parameters and the differences of the broadcast and post processed precise clock offsets [23-25]

$$
\operatorname{SISRE}=\sqrt{\left(\alpha \delta_{R}-c \delta_{T}\right)^{2}+\beta\left(\delta_{A}^{2}+\delta_{C}^{2}\right)}
$$

where $\delta_{R}, \delta_{A}$ and $\delta_{C}$ denote the errors of broadcast orbit in the radial, tangential and normal components respectively with the unit $\mathrm{m} ; \delta_{T}$ denotes the clock offset error with the unit s; $\alpha$ denotes the contribution factor of the radial component error, and $\beta$ denotes that of the tangential and normal errors. The contribution factors of different GNSS satellites are listed in Table 1.

Broadcast ionospheric model accuracy: the post-processing high-precision grid ionospheric delay model is taken as the reference model, and the accuracy of the broadcast ionospheric zenith delay value is the evaluation object. In this paper, the accuracy of BeiDou global broadcast ionospheric delay correction model (BDSGIM) is evaluated [26]. Four indexes are used for evaluation, including average bias, standard deviation, RMSE and correction percentage. The formulae are as follows $[26,27]$ :

Table 1 Average contribution factors of satellites on SISRE (elevation mask angle: $5^{\circ}$ )

\begin{tabular}{|c|c|c|c|c|}
\hline Satellite type & GEO/IC & & MEO & \\
\hline Height (km) & 35,786 & & 21,528 & \\
\hline Contribution factor & $a$ & $\beta$ & $a$ & $\beta$ \\
\hline Value & 0.9924 & 0.0867 & 0.9823 & 0.1324 \\
\hline
\end{tabular}

$$
\begin{aligned}
& \text { bias }=\frac{\sum_{i=0}^{N}\left(v T E C_{\text {model }}^{i}-v T E C_{\text {ref }}^{i}\right)}{N} \\
& s t d=\sqrt{\frac{\sum_{i=0}^{N}\left(v T E C_{\text {model }}^{i}-v T E C_{r e f}^{i}-b i a s\right)^{2}}{N-1}} \\
& R M S=\sqrt{\frac{\sum_{i=0}^{N}\left(v T E C_{\text {model }}^{i}-v T E C_{r e f}^{i}\right)^{2}}{N}} \\
& \left\{\begin{array}{l}
\operatorname{per}_{i}=\left(1-\frac{\left|v T E C_{\text {model }}^{i}-v T E C_{\text {ref }}^{i}\right|}{v T E C_{\text {ref }}^{i}}\right) \cdot 100 \% \\
\operatorname{per}=\left(\frac{\sum_{i=1}^{\left.N_{(p e r} \geq 0\right)} \text { per }_{i}}{N_{\left(\text {per }_{i} \geq 0\right)}}\right) \cdot 100 \%,\left(1-\frac{N_{\left(p r_{i} \geq 0\right)}}{N}\right) \cdot 100 \%
\end{array}\right.
\end{aligned}
$$

where $i$ denotes the $i$ th grid point; $N$ is the total number of grid points; $v T E C_{\text {model }}$ is the correction value calculated by the model at the grid point; $v T E C_{r e f}$ is the reference value.

\section{Latest performance of BDS-3}

\section{Broadcast satellite orbit accuracy}

With the continuous improvement of the BDS-3 basic constellation, the stability and connectivity of the ISL continue to increase, and the performance of the basic constellation improves gradually. The basic service performance of BDS-3 first depends on the performance of the satellite orbit and satellite clock. The performance of the BDS-3 post-processing precision orbit was estimated from the observations of international GNSS monitoring assessment service (iGMAS) stations deployed all over the world until July 2019. It indicated that the accuracies of the radial, tangential and normal components of the satellite orbit were 1.5, 5.7 and $4.1 \mathrm{~cm}$ respectively (seen in Fig. 1a), and the accuracies were $8.0,34$ and $37 \mathrm{~cm}$, respectively, when the system started offering global service in December 2018.

As shown, the post-processing orbit accuracy of the BDS-3 satellites are increased by approximately five, six and nine times, respectively. The improvement in the normal accuracy is the most significant, as the ISL observation is more abundant and stable. The accuracy of the three components (radial, tangential and normal) of the broadcast orbit of the BDS-3 by referring to the post accurate orbits are $0.059,0.323$ and $0.343 \mathrm{~m}$ respectively (seen in Fig. 1b). Compared with the launch in December 2018, the broadcast orbit has also been significantly improved. Furthermore, with the significant improvement of post-processing orbit accuracy, the 

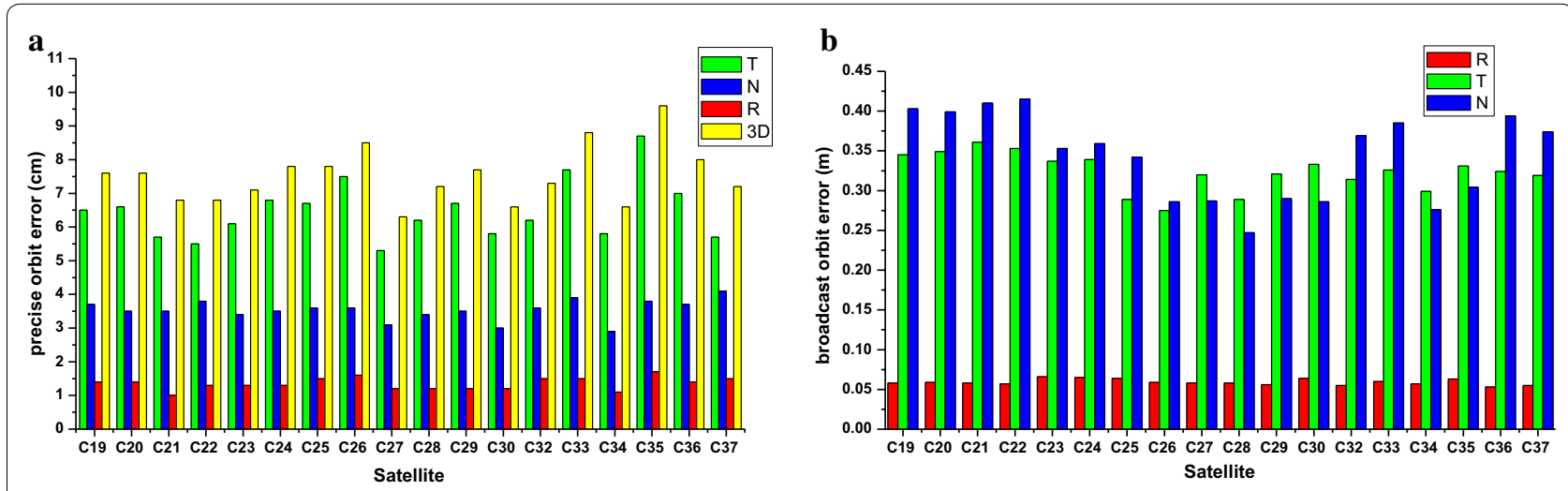

Fig. 1 a Precise orbit accuracy of the BDS-3 basic constellation. b Broadcast orbit accuracy of the BDS-3 basic constellation

estimation reliability of broadcast ephemeris based on the post-processing precise orbit has also been increased accordingly.

\section{Broadcast satellite clock offset accuracy}

The uncertainty of the broadcast satellite clock offset is a main factor affecting the user PNT service. The accuracy of the satellite broadcast clock offset is evaluated using the differences of the broadcast satellite clock offsets and post-processing precise ones. The RMS are displayed in Fig. 2.

As shown in the figure, the average uncertainty of satellite clock offset of $18 \mathrm{MEO}$ satellites is $1.55 \mathrm{~ns}$, but the satellite clock offsets of M02, M06 and M15 satellites are $3.068,2.555$ and $2.832 \mathrm{~ns}$, respectively, which are larger and unstable.

\section{Estimation of SISRE}

SISRE of the BDS-3 are estimated based on the post-processing ephemeris and broadcast ephemeris, which are shown in Fig. 3.

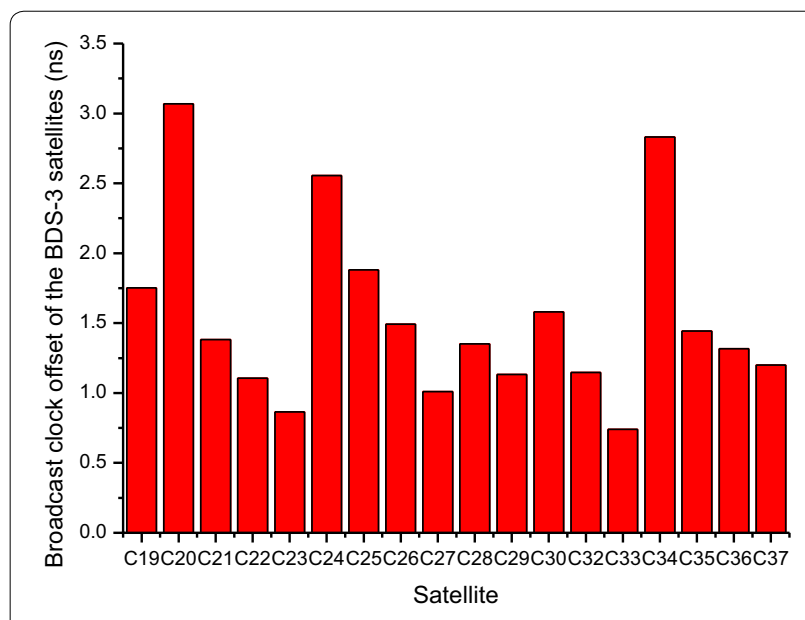

Fig. 2 Broadcast clock offset of the BDS-3 satellites

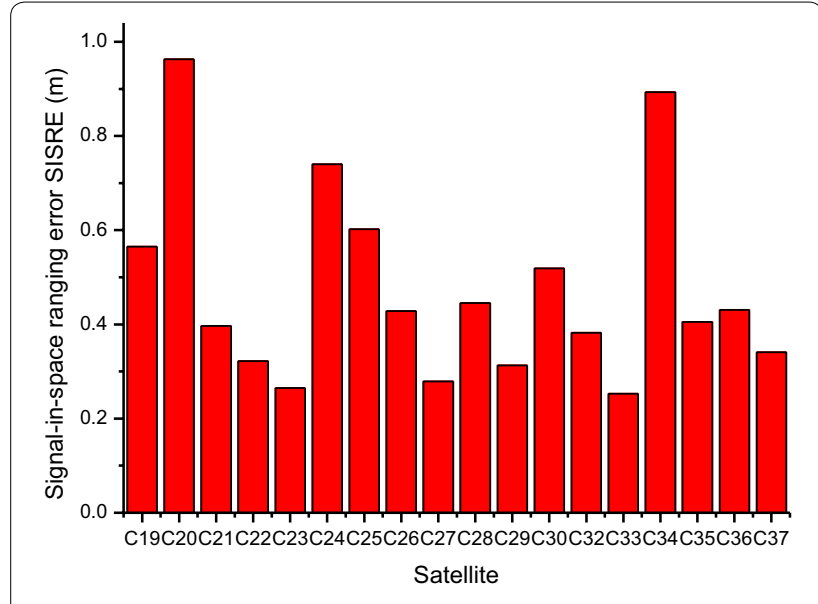

Fig. 3 SISRE of the BDS-3 satellites

Figure 3 shows that the average SISRE is approximately $0.474 \mathrm{~m}$, and all the SISREs are in agreement with the satellite clock offsets. The SISRE of the M02, M06 and M15 satellites are significantly greater than those of other satellites; when BDS-3 started offering global service in December 2018, the SISRE was approximately $0.7 \mathrm{~m}$.

\section{Accuracy of ionospheric delay correction models}

BDS-3 can broadcast four civil signals, including B1C, B1I, B2a/B2b and B3I. Among them, the B1I and B3I signals are broadcast by both BDS-2 and BDS-3. Therefore, the civil signals of BDS-3 adopt two types of ionospheric models, namely BDSK8 and BDGIM [28]. The former is used for B1I and B3I which is consistent with those of the BDS-2, while the latter is used for the new signals of BDS-3, i.e. B1a, B1C, B2a and B2b. To analyze the ionospheric correction accuracy of these two models, we used the global ionospheric maps model constructed 


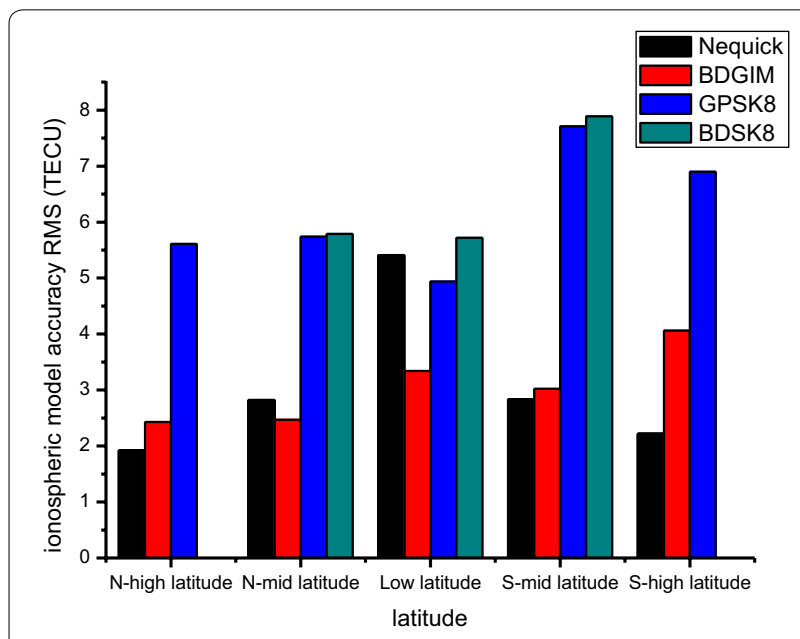

Fig. 4 Evaluation of the ionospheric model accuracy in half a year based on CODG model

by the Center for Orbit Determination in Europe (CODG model) as a reference, and compared the ninemonth (from January to September) average accuracies of Nequick, GPSK8, BDGIM and BDSK8 models. The results are shown in Fig. 4, where "high latitude" ranges from $55^{\circ}$ to $85^{\circ}$, "mid latitude" from $30^{\circ}$ to $55^{\circ}$, and "low latitudes" from $0^{\circ}$ to $30^{\circ}$.

Figure 4 shows that the correction accuracy of BDGIM model is obviously better than that of all other models in the middle and low latitude zones; in the northern middle and low latitudes, the correction ratio of BDGIM is approximately $75 \%$; in the southern middle latitude zones, the correction ratio is approximately $65 \%$; in the high latitude zones, BDGIM is slightly worse than the Nequick model; the BDGIM performs better than the GPSK8 and BDSK8 model at any latitude.
The accuracy evaluation details of BDGIM are shown in Table 2, and the correction percentage and data rejection percentage are listed in the last row of low, middle and high latitude columns.

Table 2 shows that the BDGIM maintains a good correction accuracy, regardless of the ionospheric effect.

According to the performance evaluation of the BDS-3 orbit accuracy, satellite clock offset accuracy and SISRE, the basic functions and performance of BDS-3 satisfy the design requirements.

\section{Future possible development}

Although BDS-3 has developed many new designs and functions, such as satellite orbit design, service function innovation and payload improvements [3-5], there is still room for improvements in the future.

1. The PNT service performance in high latitude area is poor. The BDS-3 constellation is composed of 24 MEO, 3 GEO and 3 IGSO satellites. The inclination of MEO and IGSO satellites is $55^{\circ}$, which indicates that the service performance of BDS- 3 will be significantly degraded in high latitude area. First, all visible BDS-3 satellites relative to the users in the polar regions are in low-elevation angle. Calculations show that the maximum altitude angle of the satellites is generally less than $50^{\circ}$ when the users are in regions of latitude greater than $85^{\circ}$ [29]. The low-elevation satellites will decrease the user ranging accuracy; next, the BDSK8 model is unavailable in the Arctic and Antarctic regions, and the accuracy of BDGIM ionospheric model is relatively low, which will degrade high-accuracy positioning. Furthermore, owing to the global climate change, sea ice in the polar regions of the Earth is melting rapidly.

Table 2 Accuracy evaluation of BeiDou global ionospheric model

\begin{tabular}{|c|c|c|c|c|}
\hline \multirow{2}{*}{$\begin{array}{l}\text { Evaluation period } \\
\text { Zones }\end{array}$} & \multicolumn{4}{|c|}{ BDGIM assessed by CODG (data from 2019.07.01 to 2019.07.31) } \\
\hline & Indicators & $\geq 15 \mathrm{TECu}$ & $<15 \mathrm{TECu}$ & Total \\
\hline \multirow[t]{4}{*}{ Low latitude } & Bias/TECu & -5.45 & -2.56 & -3.35 \\
\hline & STD/TECU & 2.75 & 2.21 & 2.70 \\
\hline & RMS/TECu & 6.11 & 3.38 & 4.31 \\
\hline & Per/\% & $(72.45,0.00)$ & $(60.97,0.23)$ & $(64.13,0.16)$ \\
\hline \multirow[t]{4}{*}{ Middle latitude } & Bias/TECu & -4.11 & -2.16 & -2.24 \\
\hline & STD/TECU & 2.31 & 2.35 & 2.38 \\
\hline & RMS/TECu & 4.72 & 3.20 & 3.27 \\
\hline & Per/\% & $(75.48,0.00)$ & $(65.06,0.70)$ & $(65.45,0.67)$ \\
\hline \multirow[t]{4}{*}{ High latitude } & Bias/TECu & -4.03 & -1.18 & -1.18 \\
\hline & STD/TECu & 0.55 & 1.54 & 1.54 \\
\hline & RMS/TECu & 4.06 & 1.95 & 1.95 \\
\hline & Per/\% & $(74.53,0.00)$ & $(69.63,1.79)$ & $(69.63,1.79)$ \\
\hline
\end{tabular}


Table 3 Statistics of mean elevation angle with changes of the IGSO inclinations

\begin{tabular}{llll}
\hline IGSO inclination $\left({ }^{\circ}\right)$ & Range & Mean value $\left({ }^{\circ}\right)$ & $\operatorname{Max}\left({ }^{\circ}\right)$ \\
\hline 55 & High latitude & 31.52 & 66.71 \\
65 & $75^{\circ}-90^{\circ} \mathrm{N}$ & 37.13 & 78.39 \\
75 & & 41.87 & 89.94 \\
\hline
\end{tabular}

Especially after ice and snow in the Arctic has melted in the summer, the demand for PNT service in the Arctic region becomes more urgent considering the significant route value and rich resource reserve $[29$, 30].

To ensure the efficiency of all kinds of scientific research and the safety of transportation in the Arctic region, the satellite navigation service must be improved. For BDS-3, a reasonable and simplified method is to increase the inclination of IGSO satellites.

Using the actual constellation of BDS-3, we analyzed the user's elevation angle variation according to changes in the orbit inclination of the IGSO satellites in highlatitude areas by adjusting the inclination of the IGSO constellation to $55^{\circ}, 65^{\circ}$ and $75^{\circ}$. Seven-day simulation data with a sampling interval of $10 \mathrm{~min}$ were used. To calculate the average dilution of precision (DOP), grids with the longitude interval of $2^{\circ}$ and latitude interval of $1^{\circ}$ were used, and the minimum altitude angle was set as $5^{\circ}$. The latitude of key test area ranged from $75^{\circ}$ to $90^{\circ}(\mathrm{N})$ whereas the longitude ranged from $-180^{\circ}$ to $180^{\circ}$.

Table 3 shows that with a $10^{\circ}$ increase of the inclination angle of the IGSO satellites in the high latitude region, the average altitude angle of the visible satellite increases by approximately $5^{\circ}$, and the maximum altitude angle increases by approximately $12^{\circ}$. With the increase in satellite elevation angle, the number of visible satellites will increase, and the effect of ionosphere will be weakened.

2. An obvious "south wall effect" exists in BDSPPP and regional short message communication (RSMC) services based on GEO satellites. For users in the northern hemisphere, GEO satellites are always located south of the user, and for those at higher latitudes, the BDSPPP and short message communication service will likely be interrupted once obstacles appear in the south. The "south wall effect" is particularly serious for users of PPP, as $20-30 \mathrm{~min}$ are required to obtain the converged results after the PPP service recovers from the GEO satellites. Furthermore, the repeated convergence will result in a significant reduction in the application efficiency of BDSPPP in cities with high-rise buildings. Similarly, users in southern hemisphere will experience the "north wall effect".

To overcome the "south wall effect" of BDSPPP and RSMC provided by BeiDou GEO satellites, the most effective method is to use the BeiDou IGSO satellites to transmit rapid precise orbit and clock offset parameters, as well as the regional short message. IGSO and GEO satellites working together, can eliminate the "south wall effect" and effectively improve the featured service efficiency of BDS. Furthermore, if the inclination angle of IGSO satellites are increased and an appropriate ground reference station is built in the Arctic region, then the BDSPPP and RSMC services can be provided to users in the Arctic region.

3. Some satellites' services are interrupted during the orbit maneuver of the satellites. Satellite orbit maneuver is inevitable. Almost every month, BeiDou GEO satellite must adjust its East-West orbit, and conduct a North-South orbit maneuver every half a year. IGSO and MEO satellites generally conduct an orbit maneuver every half a year. During the period of orbit adjustment, the satellite ephemeris cannot reflect the orbit maneuver quickly and is typically expressed as "unavailable" or "unhealthy". Furthermore, the PNT service of the corresponding satellite will be suspended. The duration is approximately $7 \mathrm{~h}$. For IGSO and MEO satellites, owing to their large number, low frequencies of adjustment and the sole functions, the PNT service will not be significantly affected in general. However, the BDSPPP and RSMC service provided by the three GEO satellites of the BDS-3 will significantly affect many authorized users owing to the unavailability caused by GEO satellites' orbit maneuver.

As we know, the maneuvering satellites cannot provide normal service because the satellite ephemeris cannot accurately reflect the actual positions of the satellites. If inertial navigation system (INS) equipment was installed on the satellite, the orbit change in the satellite maneuver could be measured during the satellite maneuver, and thus the orbit parameters of the moving satellites could be obtained. However, one of the main problems is that during the nonmaneuverable period of the satellite, the on-board INS must be calibrated using a precise satellite orbit or an error correction model must be established. Once the satellite enters the maneuvering state, INS observations and corresponding corrections can be used to provide the satellite position during the maneuvering period, which will improve the availability of the maneuvering satellites. 
4. Datum drift and rotation of BeiDou satellite constellation will occur in autonomous navigation. If only ISL measurements are used in the autonomous navigation, then only the relative positions of constellation can be solidified. The overall drifts of the spatial datum and time datum cannot be determined or solidified, and neither can the overall rotation of the entire constellation. These types of systematic errors may not significantly affect high-accuracy differential positioning users; however, they will significantly affect the real-time navigation and timing service users.

Regarding the drift and rotation of space and time reference of the BeiDou constellation in autonomous navigation, we may use high-accuracy and high stable hydrogen clocks or cesium clocks onboard as references, and then the autonomous time keeping of satellite clocks can be realized to reduce the time drift of the entire constellation. In addition, the onboard GNSS receiver can receive the signals of other GNSS satellites and determine the onboard satellite orbit; thus, the high-precision orbits of BeiDou satellites can be determined based on the joint adjustment with the autonomous orbit determination data. Therefore, the space and time references of the BeiDou satellite constellation can be maintained, and the datum drift and rotation of the autonomous orbit determination can be reduced.

5. Inconsistency of the BDS-3 ionospheric model. As previously analyzed, the B1I and B3I signals of BDS-2 are maintained in BDS-3, and the corresponding ionospheric model adopts the BDSK8 model. However, new signals such as $\mathrm{B} 1 \mathrm{C}, \mathrm{B} 2 \mathrm{a}$ and $\mathrm{B} 2 \mathrm{~b}$ use the BDGIM ionospheric correction model. Different models result in the inconsistent of accuracies, which not only causes confusion among terminal manufacturers and users, but also reduces the ranging accuracy of B1I and B3I.

Regarding the inconsistency of the BDS-3 ionospheric models, using the BDGIM as the unified model of all signals of the BDS-3 can reduce the confusion and inconvenience in users and terminal manufacturers. Since the accuracy of BDGIM model [28] is higher than that of BDSK8, the user equivalent range error can be improved slightly after the unification. Considering that BDS-2 is close to being obsolete, and to not affect the development of the BDS-3 terminal and the PNT service performance of BDS-3 users, we suggest unifying the ionospheric models for BDS-3 the soonest possible time for reducing loss to users and receiver manufacturers. In addition, the ionospheric delay correction model in high-latitude areas should be refined.
6. Vulnerability exists inherently in satellite navigation systems, such as the satellite constellation, the ground operational control system (OCS) and the signals. Once the core subsystem fails or malfunctions, e.g., power interruption, time system failure, and other core equipment failures, the PNT service may be interrupted.

To resolve the vulnerability of the BDS constellation, OCS and signals, we can expand PNT information sources by building a comprehensive PNT system [3133], and then use the resilient PNT theoretical framework [34] to realize the resilient integration of multiple PNT sensors, and build the resilient function model, the resilient stochastic model, and resilient data fusion methodology, with the aim of realizing a seamless PNT service from deep space to deep sea, and from outdoor to indoor.

\section{Conclusions}

BDS-3 satisfied the requirements of design indexes in orbit determination accuracy, satellite clock accuracy, signal-in-space accuracy and PNT service performance. Additionally, the featured services such as BDSPPP, BDSBAS, regional message communication, and the global SAR function have wide application prospects.

In the future, the inclination angle of IGSO satellites may be increased to improve the service performance of BDS in polar regions. The IGSO satellites may be designed for providing the RSMC and BDSPPP services to overcome the "south wall effects". The INS payloads are suggested to be added to various satellites, and thus the continuity and availability of satellite orbit parameters can be guaranteed in satellite maneuver. The high accuracy hydrogen clocks or cesium atomic clocks onboard might be used to control the time drift of satellite constellation during the autonomous navigation with the support of ISLs. The two commonly used BDS ionospheric models should be unified for all signals to reduce the users' confusion and improve the range accuracy for the B1I and B3I signals. The comprehensive and resilient PNT infrastructure should be established for the seamless PNT services.

\section{Acknowledgements \\ We are immensely grateful to the BeiDou Navigation Satellite Project Develop- ment Team for years of hard work and all major breakthroughs they have achieved in a series of core technologies represented by constellation design, inter-satellite links and the expansion of featured service. We would also like to thank our colleagues from the State Key Laboratory of Geo-Information Engi- neering who provided insight and expertise that greatly assisted the research.}

\section{Authors' contributions}

YY proposed the idea and drafted the article; YM carried out the evaluation and assisted in data analysis; BS assisted in data collection and article revision.

All authors read and approved the final manuscript. 


\section{Funding}

This work was supported by the National Natural Science Foundation of China (Grant No. 41931076) and the National Key Technologies R\&D Program of China (Grant No. 2016YFB0501700).

\section{Availability of data and materials}

The datasets analyzed during the current study are available in the data repositories of Test and Assessment Research Center of China Satellite Navigation Office (www.csno-tarc.cn) and the Crustal Dynamics Data Information System of NASA (ftp://cddis.nasa.gov/gnss/products/ionex).

\section{Competing interests}

The authors declare that they have no competing interests.

Received: 14 November 2019 Accepted: 23 December 2019

Published online: 20 January 2020

\section{References}

1. Yang, Y. X., Tang, J., \& Montenbruck, O. (2017). Chinese satellite navigation system. In P. Teunissen \& O. Montenbruck (Eds.), Handbook of global navigation satellite system (pp. 273-304). New York: Springer.

2. Yang, Y. X., Xu, Y., Li, J., \& Yang, C. (2018). Progress and performance evaluation of BeiDou global navigation satellite system: Data analysis based on BDS-3 demonstration system. Science China Earth Sciences, 61(5), 614-624.

3. Yang, Y. F., Yang, Y. X., Hu, X., Chen, J., Guo, R., Tang, C., et al. (2019). Intersatellite link enhanced orbit determination for BeiDou-3. Navigation, 66(1), 1-16. https://doi.org/10.1017/S0373463319000523.

4. Yang, Y. X., Gao, W., Guo, S., Mao, Y., \& Yang, Y. (2019). Introduction to BeiDou-3 navigation satellite system. Navigation, 66(1), 7-18.

5. Yang, Y. X., Yang, Y., Hu, X., Tang, C., Zhao, L., \& Xu, J. (2019). Comparison and analysis of two orbit determination methods for BDS-3 satellites. Acta Geodaetica et Cartographica Sinica, 48(7), 831-839. https://doi.org/10.11947 /j.AGCS.2019.20180560.

6. Liu, L., \& Zhang, T. (2019). Improved design of operational system in BDS-3. Navigation, 66(1), 37-47.

7. Guo, S., Cai, H., Meng, Y., Geng, C., Jia, X., Mao, Y., et al. (2019). BDS-3 RNSS technical characteristics and service performance. Acta Geodaetica et Cartographica Sinica, 48(7), 820-821. https://doi.org/10.11947/j.AGCS.2019.20190 091.

8. Xie, X., Geng, T., Zhao, Q., Liu, J., \& Wang, B. (2017). Performance of BDS-3: Measurement quality analysis, precise orbit and clock determination. Sensors, 17(6), 1233. https://doi.org/10.3390/s17061233.

9. Xie, X., Geng, T., Zhao, Q., Cai, H., Zhang, F., Wang, X., et al. (2019). Precise orbit determination for BDS-3 satellites using satellite-ground and inter-satellite link observations. GPS Solutions, 23(2), 40. https://doi.org/10.1007/s1029 1-019-0823-5.

10. Xu, X., Wang, X., Liu, J., \& Zhao, Q. (2019). Characteristics of BDS3 global service satellites: $P O D$, open service signal and atomic clock performance. Remote Sensing, 11(13), 1559. https://doi.org/10.3390/rs11131559.

11. Ren, X., Yang, Y., Zhu, J., \& Xu, T. (2017). Orbit determination of the nextgeneration BeiDou satellites with intersatellite link measurements and a priori orbit constraints. Advances in Space Research, 60(10), 2155-2165. https ://doi.org/10.1016/j.asr.2017.08.024

12. Ren, X., Yang, Y., Zhu, J., \& Xu, T. (2019). Comparing satellite orbit determination by batch processing and extended Kalman filtering using inter-satellite link measurements of the next-generation BeiDou satellites. GPS Solutions, 23, 25. https://doi.org/10.1007/s10291-018-0816-9.

13. Yang, Y. X., \& Ren, X. (2018). The maintenance of space datum for autonomous satellite navigation. Geomatics and Information Science of Wuhan University, 43(12), 1780-1786.

14. Lu, M., Li, W., Yao, Z., \& Cui, X. (2019). Overview of BDS III new signals. Navigation, 66(1), 19-35.

15. Wang, C., Zhao, Q., Guo, J., Liu, J., \& Chen, G. (2019). The contribution of inter-satellite links to BDS-3 orbit determination: Model refinement and comparisons. Navigation, 66(1), 71-82.
16. Li, X. (2018). Triple frequency PPP ambiguity resolution with BDS2 and BDS3 observations. In 31st International technical meeting of the satellite division of the institute of navigation (ION GNSS+ 2018) (pp. 3833-3858), Miami, Florida.

17. Yang, Y. X. (2009). Chinese geodetic coordinate system 2000. Chinese Science Bulletin, 54(16), 2714-2721.

18. Han, C., Yang, Y., \& Cai, Z. (2011). BeiDou navigation satellite system and its time scales. Metrologia, 48(4), 1-6.

19. China Satellite Navigation Office. (2016). BeiDou navigation satellite system signal in space interface control document: Open service signal (version 2.1). Retrieved October 20, 2019, from http://www.beidou.gov.cn/xt/ gfxz/201805/P020180507527106075323.pdf.

20. China Satellite Navigation Office. (2017). BeiDou navigation satellite system signal in space interface control document: Open service signal B1C (version 1.0). Retrieved October 20, 2019, from http://www.beidou.gov.cn/xt/ gfxz/201712/P020171226741342013031.pdf.

21. China Satellite Navigation Office. (2017). BeiDou navigation satellite system signal in space interface control document: Open service signal B2a (version 1.0). Retrieved October 20, 2019, from http://www.beidou.gov.cn/xt/ gfxz/201712/P020171226742357364174.pdf.

22. China Satellite Navigation Office. (2018). BeiDou navigation satellite system signal in space interface control document: Open service signal B3I (version 1.0). Retrieved October 20, 2019, from http://www.beidou.gov.cn/xt/ gfxz/201802/P020180209623601401189.pdf.

23. Hu, Z. (2013). BeiDou navigation satellite system performance assessment theory and experimental verification. Doctoral Dissertation, Wuhan University, Wuhan

24. Spilker, J. J., Jr., Axelrad, P., Parkinson, B., \& Enge, P. (1994). Global positioning system: Theory and application volume I (p. 1994). Stanford: American Institute of Aeronautics and Astronautics.

25. U.S. Department of Defense. (2008). Global positioning system standard positioning service performance standard (4th ed). Retrieved September 18 2008, fromhttps://www.gps.gov/technical/ps/2008-SPS-performance-stand ard.pdf.

26. Wang, N., Yuan, Y., Li, Z., Li, M., \& Huo, X. (2017). Performance analysis of different NeQuick ionospheric model parameters. Acta Geodaetic et Cartographica Sinica, 46(4), 421-429.

27. Zhang, Q., Zhao, Q. L., Zhang, H. P., Hu, Z. G., \&Wu, Y. (2014). Evaluation on the precision of Klobuchar model for BeiDou navigation satellite system. Geomatics and Information Science of Wuhan University, 39(2), 142-146.

28. Yuan, Y., Wang, N., Li, Z., \& Huo, X. (2019). The BeiDou globally broadcast ionospheric delay correction model (BDGIM) and its preliminary test. Navigation, 66(1), 55-69.

29. Peng, H., Yang, Y., Wang, G., \& He, H. (2016). Performance analysis of BDS satellite orbits during eclipse periods: Results of satellite laser ranging validation. Acta Geodaetica et Cartographica Sinica, 45(6), 639-645. https://doi. org/10.11947/j.AGCS.2016.20150637.

30. Yang, Y. X., \& Xu, J. (2016). Navigation performance of Beidou in polar area. Geomatics and Information Science of Wuhan University, 41(1), 15-20.

31. Li, J., Yang, Y., He, H., \& Guo, H. (2017). An analytical study on the carrier-phase linear combinations for triple-frequency GNSS. Journal of Geodesy, 91(2), 151-166. https://doi.org/10.1007/s00190-016-0945-2.

32. Yang, Y. X. (2016). Concepts of comprehensive PNT and related key technologies. Acta Geodaetica et Cartographic Sinica, 45(5), 505-510.

33. Yang, Y. X., \& Li, X. (2016). Micro-PNT and comprehensive PNT. Acta Geodaetica et Cartographic Sinica, 45(10), 1249-1254.

34. Yang, Y. X. (2018). Resilient PNT concept frame. Acta Geodaetica et Cartographica Sinica, 47(07), 893-898.

\section{Publisher's Note}

Springer Nature remains neutral with regard to jurisdictional claims in published maps and institutional affiliations. 